\title{
Characteristics of patients hospitalised in an emergency department observation unit in Japan
}

\author{
Toshiya Mitsunaga, Yuhei Ohtaki, Nobuaki Kiriyama, Kei Ohtani, Wataru Yajima, \\ Takahiko Hibi, Satoshi Takeda \\ Department of Emergency Medicine, Jikei University School of Medicine, Minato-ku, Tokyo, Japan
}

\begin{abstract}
In Japan, transporting elderly patients to emergency departments has recently posed serious problems, including a longer average time from patients' initial emergency calls to their arrival at hospitals. To manage emergency departments more efficiently, many hospitals in the United States and some other developed countries, including Japan, introduced emergency department observation units (EDOU). However, because the usefulness of EDOUs in managing elderly patients remains uncertain, we analysed data of patients admitted to a Japanese university hospital's EDOU to gauge its efficacy. We followed 1,426 patients admitted to the hospital's EDOU from 1 January 2011 to 31 December 2014. The average age of patients who stayed in the EDOU increased, whereas the average length of time that they spent there decreased. Although the percentage of patients older than 65 years increased slightly, from $36.42 \%$ (2011-2012) to $37.73 \%$ (2013-2014), the proportion of those patients between the two periods did not significantly change $(\mathrm{P}=.61)$. Moreover, their average length of stay was $2.16 \pm 0.91$ days, whereas patients younger than 65 years stayed for significantly less time (1.92 \pm 0.82 days). By condition,
\end{abstract}

\footnotetext{
Correspondence: Toshiya Mitsunaga, Department of Emergency Medicine, Jikei University School of Medicine, 3-25-8 Nishi-Shinbashi, Minato-ku, Tokyo 105-8461, Japan.

Tel.: +81.3.3433.1111 - Fax: +81.3.5400.1252.

E-mail: toshiya.m@jikei.ac.jp

Key words: Observation unit; Emergency medicine; Japan, University hospital; Elderly patients.

Contributions: TM was responsible for data processing and drafting the manuscript; NK, KO, WY, and TH collected data; and KO and ST critically evaluated the process.

Conflict of interest: the authors declare no potential conflict of interest.

Funding: none.

Received for publication: 28 February 2018.

Revision received: 13 April 2018.

Accepted for publication: 18 April 2018.

This work is licensed under a Creative Commons Attribution 4.0 License (by-nc 4.0).

(C) Copyright T. Mitsunaga et al., 2018

Licensee PAGEPress, Italy

Emergency Care Journal 2018; 14:7381

doi:10.4081/ecj.2018.7381
}

approximately $36 \%$ of patients older than 65 years presented with non-neurosurgical trauma, approximately 59\% presented with other forms of trauma, but proportions of both categories of trauma were significantly smaller in patients younger than 65 years (nonneurosurgical trauma, 23\%; all trauma, 47\%). Most elderly patients with limb trauma prepped for surgery were transferred to other hospitals after a few days. Results suggest that the EDOU at the university hospital has served to as a buffer for regional emergency medical systems in Japan, especially given the continued ageing of the Japanese population.

\section{Introduction}

In Japan, delays in emergency transport to hospitals due to hospitals' refusal to accept patients seeking emergency care have become a serious problem, especially in Tokyo. In 2012 the average time from patients' initial emergency calls to their arrival at hospitals was 38.7 minutes nationwide, whereas in Tokyo Prefecture it was 54.9 minutes - the longest in Japan. ${ }^{1}$ Transport times to hospitals have moreover increased with each passing year. Among their reasons to refuse patients seeking emergency care, hospitals not only in Japan but also in developed and developing countries often cite overcrowding in their emergency departments. ${ }^{2-4}$

\section{Japan's emergency system}

In Japan, emergency departments nationwide are grouped into three categories. Primary emergency medical institutions, totalling 1,376 in number, treat patients with mild conditions and walk-in patients. By contrast, and by far the most common, secondary emergency medical institutions, totalling 3,865 , treat patients with mild or moderate conditions that might require hospitalisation. Last, totalling 270, Japan's relatively few tertiary emergency medical institutions treat or resuscitate seriously ill patients, including ones who have suffered from multiple trauma or cardiopulmonary arrest. ${ }^{1}$ Although data about the number of patients transported to the three kinds of institution are unavailable, data about patients' severity show that $50.4 \%$ of cases were mild, $38.9 \%$ were moderate and only $9.1 \%$ were severe. ${ }^{1}$ In Japan, when a person is injured or suffering from disease, he or she or someone nearby calls 119 , the emergency telephone line managed by local fire departments. Staff members of the emergency line, all of whom work for local fire departments, gather information about the person's situation and contact the fire station closest to the scene. Emergency medical response is immediately activated, and the paramedic team travels to the scene by ambulance. Upon meeting the person seeking care, the team gathers his or her biological information or acknowledges his or her injury and determines the triage level, according to which the person, now a patient, is transferred to a primary, secondary or tertiary emergency medical institution.

As of late 2012, 4,965 paramedic teams were on call in Japan. ${ }^{1}$ 
Each team consists of firefighters working for emergency medical services and emergency medical technicians, which in 2012 formed the teams in proportions of $61.68 \%$ and $38.32 \%$, respectively. ${ }^{1}$ Although nearly all $(95.9 \%)$ paramedic teams that year consisted of emergency medical technicians, $4.1 \%$ did not. ${ }^{1}$ In general, paramedic teams are specially trained to transfer patients to hospitals, though fire laws limit what the teams' emergency medical technicians delivering treatment can do for patients. Some operations can be performed under the direction of doctors (e.g. securing the airway, respiratory support, fixation for trauma, sugar administration for hypoglycaemia, rapid infusion for shock and epinephrine administration and defibrillation for cardiopulmonary arrest). ${ }^{1}$

Although typically required to accept all patients, emergency medical institutions in Japan, especially tertiary ones, cannot always accept patients, even if they are in critical condition. Some institutions simply lack the facilities to treat seriously ill patients, while others cannot accept patients in certain departments, their initial treatment beds or hospital beds are entirely occupied or their operating theatres lack capacity. In Japan, the unchanged number of secondary emergency medical institutions despite the increasing number of elderly patients, especially ones older than 85 years 912,120 in 2014 and 968,442 in 2015 - are major causes of overcrowding in emergency departments. ${ }^{5-7}$ Japan's burgeoning elderly population particularly frustrates the management of emergency departments, ${ }^{6}$ because elderly patients often require long-term hospitalisation, and diagnosing their conditions can be quite difficult when their chief complaints are vague. In Japan, the proportion of people older than 65 years was $23.0 \%$ in 2010 - the highest in the world - and is expected to reach $29.1 \%$ by 2020 , compared to projections of $19.4 \%$ by 2020 in developed countries worldwide. ${ }^{8}$

\section{The Tokyo rule}

In 2009, to manage so-called difficult cases of transport to emergency medical institutions, Tokyo Prefecture introduced the Tokyo Rule. The rule pertains particularly to sub-moderate cases and patients whose destinations remain undetermined after 20 minutes though emergency medical services have already contacted at least five hospitals. If the case is subject to the Tokyo Rule, then emergency medical services contact one of Tokyo's 80 regional emergency medical centres. ${ }^{9}$ If the centre cannot accept the patient, then the centre's emergency department manager and the emergency medical service seek out a hospital in the region that can accept the patient. If they cannot recruit a hospital in the region, then they contact an emergency medical coordinator at the Tokyo Fire Department, who will contact other regional emergency medical centres for a hospital that can accept the patient. ${ }^{9}$ In 2011, difficult cases of transport totalled 14,449 , which was roughly a third of the total in $2008(35,746){ }^{9}$

Although adherence to the Tokyo Rule affords some benefits, its overall effects have been limited. ${ }^{9}$ The keywords of difficult cases of transport have been not only orthopaedics, alcohol and mental illness but also elderly patient, which indicates that the problems of elderly patients in obtaining transport to emergency medical facilities in Japan persist. ${ }^{9}$

\section{Emergency department observation unit}

In the United States and some other developed countries, where the massive number of patients who present at emergency departments has also become a serious problem, ${ }^{10,11}$ hospitals have introduced emergency department observation units (EDOU) in order to not only manage emergency departments safely and smoothly but also reduce the number of cases requiring hospitali- sation. ${ }^{12}$ Although many hospitals in Japan have introduced EDOUs as well, their usefulness in managing elderly patients remains unclear. In response, we retrospectively analysed the data of patients admitted to a university hospital's EDOU from 2011 to 2014 in order to examine the EDOU's role in managing elderly patients.

\section{Materials and Methods}

\section{The university hospital and its emergency department}

The university hospital's emergency department that we examined is a secondary emergency medical institution in central Tokyo with 1,075 beds and affiliated with Jikei University School of Medicine. The characteristics of the emergency department are similar to those of emergency rooms in North America; it has seven beds for initial treatment and six examination rooms for services on holidays and at night. Annually, the department receives approximately 25,000 patients and 8,000-8,500 ambulance deliveries. All patients who present at the emergency department are first seen by emergency physicians who consult doctors in other departments as necessary. All care after hospitalisation is managed by doctors outside the emergency department.

\section{The hospital's emergency department observation units}

The university hospital has one EDOU with 14 beds. Patients are observed in the EDOU for a maximum of 72 hours, after which they may be discharged, transferred to another department or another hospital, or sent home. All care administered during stays in the EDOU is managed by physicians and nurses in the emergency department. The vital signs of all patients are monitored by biometric information devices that form part of a patient information system (IntelliVue, Philips).

\section{Protocol for admission to the emergency department observation units}

Patients are admitted to the EDOU in three situations: i) When operating theatres lack capacity and the theatres' schedules cannot be rearranged, in which cases the emergency department is the patient's primary department and its staff organise the transfer of patients to other hospitals; ii) When stays are expected to be brief due to the nature of the case (e.g. acute intoxication, acute low back pain, syncope, headache, peripheral vertigo, asthma attack, anaphylaxis and minor injuries such as sprains and bruises); or iii) In cases in which, for example, patients are too old to return home by themselves, they visit the department at midnight and cannot return home due to lack of available transport or the diagnosis remains unclear but serious pathology (e.g. acute myocardial infarction and stroke) cannot be ruled out.

\section{Data collection}

From 1 January 2011 to 31 December 2014, 1,734 patients were admitted to the EDOU under the care of the emergency department. They formed the study sample. After we excluded 308 patients from analysis due to insufficient data, we analysed the data of 1,426 patients, whom we divided into two groups; Group A consisted of 615 patients who stayed in the EDOU from 1 January 2011 to 31 December 2012, whereas Group B consisted of 811 patients who stayed there from 1 January 2013 to 31 December 2014 (Figure 1). 


\section{Classification of diseases and injuries}

We classified diseases and injuries listed in the medical record into seven groups: i) Non-neurosurgical trauma, including trauma other than head trauma with fractures, bruising, signs of abuse or haematoma but excluding cervical spine lesions; ii) Neurosurgical trauma, including cervical spine lesions and head trauma with fractures, bruising, signs of abuse or intracranial bleeding; iii) Acute intoxication; iv) Accidental ingestion (e.g. of foreign bodies and chemicals); v) Environmentally induced conditions (e.g. heatstroke); vi) Psychiatric disease; and vii) Internal medicine diseases.

\section{Statistical analyses}

For the mentioned patient groups, we examined the average age, sex ratio, average length of stay in the EDOU, the proportions of patients older than 65 and 85 years, the classification of diseases and injuries, the relationship between age and average length of stay in the EDOU and the rate of coexisting medical problems in patients who had suffered from trauma. We recorded quantitative variables as $M \pm S D$ and qualitative variables as percentages. We used a Student's $t$ test to compare variables for average age, average length of stay and the relationship between age and average length of stay, whereas we used a chi-square test to compare variables for sex ratio, the proportions of patients older than 65 and 85 years, the classification of diseases and injuries at admission and the rate of coexisting medical problems in patients with trauma. A P-value less than .05 was considered to indicate statistical significance.

\section{Results}

i) Average age; ii) Sex ratio; iii) Average length of stay in the EDOU: the background information of patients in the sample appears in Table 1. Between the groups (i.e., over time), the gender ratio did not change, the average age slightly increased and the average length of stay in the EDOU lessened. However, none of those changes were significant; iv) Proportions of patients older than 65 and 85 years. As shown in Figure 2, the proportion of patients older than 65 years slightly increased from $36.42 \%$ (Group A) to $37.73 \%$ (Group B), although not significantly $(\mathrm{P}=.61)$. By contrast, the proportion of patients older than 85 years significantly $(\mathrm{P}=.012$ ) increased from $6.99 \%$ (Group A) to $10.85 \%$ (Group B); v) Classification of diseases and injuries: as shown in Figure 3, among patients older than 65 years, the percentage of cases of nonneurosurgical trauma was highest (36\%) and significantly higher than that of patients younger than 65 years. Also among those patients, the proportion of trauma cases, including neurosurgical trauma, accounted for approximately $60 \%$ of all diseases and injuries, which was significantly higher than that of patients younger than 65 years. Conversely, in the group of patients less than 65 years old, the proportion of acute intoxication was significantly higher; vi) Relationship between age and average length of stay in the EDOU. As shown in Figure 4, the average length of stay in the EDOU of patients older than 65 years was $2.16 \pm 0.91$ days, which was significantly longer than that of patients younger than 65 years ( $1.92 \pm 0.82$ days). For both groups, the maximum length of stay in the EDOU was 6 days, and the minimum length was 1 day; vii) Proportion of patients discharged, transferred to another

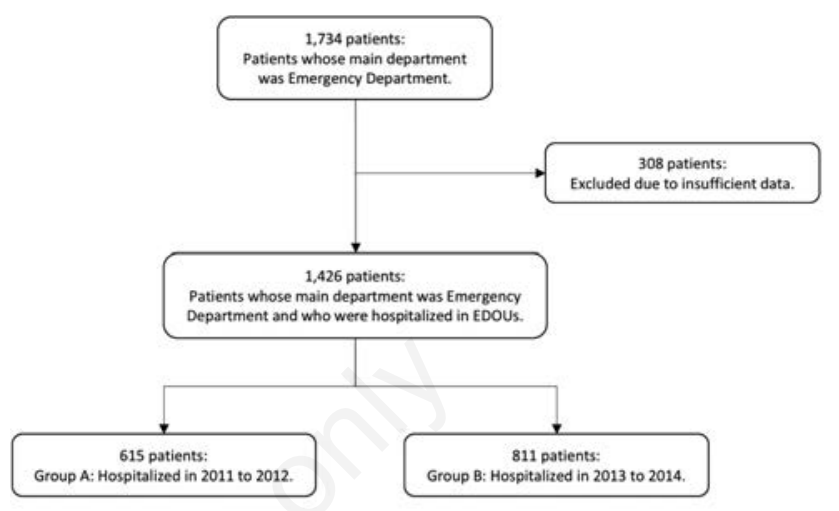

Figure 1. Flowchart of sample selection.

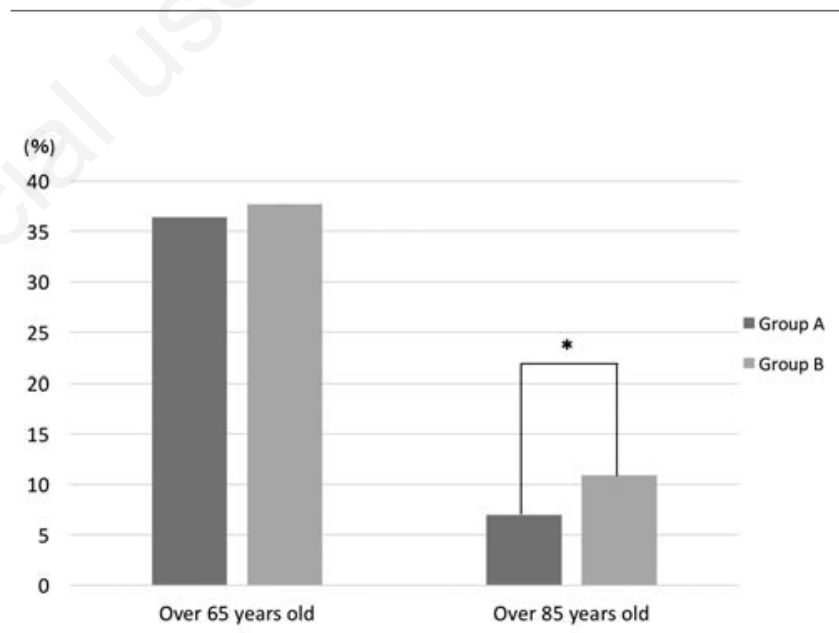

Figure 2. Proportion of patients older than 65 and 85 years who stayed in the university hospital's emergency department observation units (EDOU). Group A consisted of patients who stayed in the EDOU from 1 January 2011 to 31 December 2012, whereas Group B consisted of patients who stayed there from 1 January 2013 to 31 December 2014. We analysed data with a chi-square test $(* P<.05)$.

Table 1. Background information of patients in the sample. Values appear as $M \pm S D$ and percentages. We analysed data regarding average age and average length of stay with a Student's $t$ test and sex ratio with a chi-square test. Group A consisted of patients who stayed in the emergency department observation units from 1 January 2011 to 31 December 2012, whereas Group B consisted of patients who stayed there from 1 January 2013 to 31 December 2014.

\begin{tabular}{lcccc} 
& Group A $(\mathbf{n}=615)$ & Group B (n=811) & P \\
Average age (in years) & $53.82 \pm 21.62$ & $54.84 \pm 23.02$ & .39 \\
Sex ratio & Male: $387(62.93 \%)$ & Male: $482(59.43 \%)$ & .18 \\
\hline Average length of stay in the EDOU (in days) & Female: $228(37.07 \%)$ & Female: $329(40.57 \%)$ & .26 \\
\hline
\end{tabular}


department or transferred to another hospital. Patients who were discharged, transferred to another department or transferred to another hospital totalled 955 (67\%), 197 (14\%) and 274 (19\%), respectively. As shown in Figure 5, the proportion of cases of nonneurosurgical trauma was greater among patients transferred to another hospital, while 40 patients $(56.3 \%)$ needed semi-urgent operation; viii) Rate of coexisting medical problems in patients with trauma. As shown in Figure 6, the rate of coexisting medical problems in patients younger than 65 years who stayed in the EDOU for trauma was $4.99 \%$, which was significantly lower than that of patients older than 65 years.

\section{Discussion}

According to Yoshida et al. (2016), ${ }^{13}$ among patients hospitalised in emergency medical institutions in Japan, 58.7\% were older than 65 years. In our study, such patients accounted for roughly $35 \%$ of the sample and increased in number over time. In

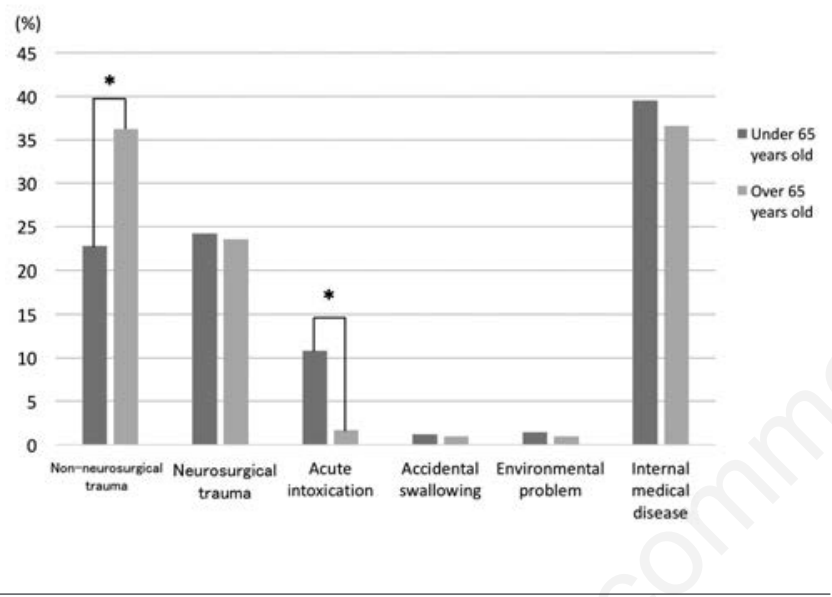

Figure 3. Details of the classification of diseases and injuries among patients admitted to the university hospital's emergency department observation units. We analysed data with a chisquare test $(* \mathrm{P}<$.05).

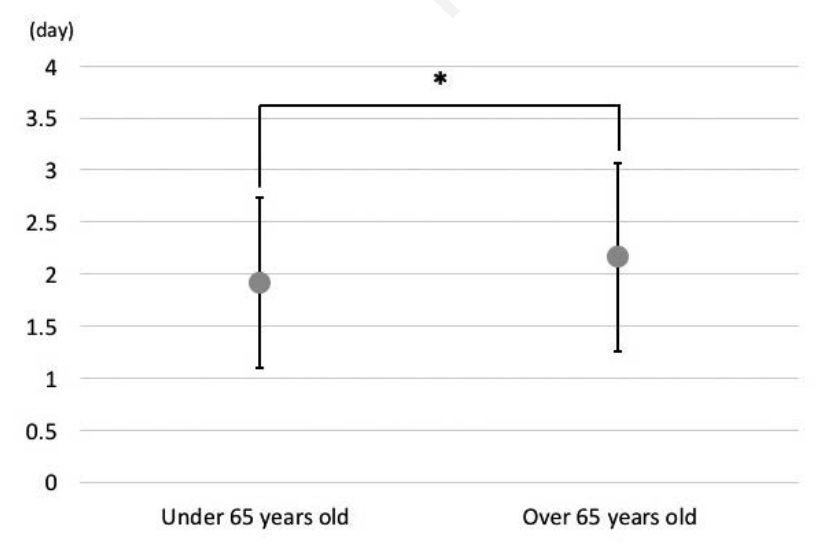

Figure 4. Relationship between age and average length of stay in the emergency department observation units. Data appear as $M$ \pm SD determined with a Student's t test $(\star P<.05)$. particular, the proportion of patients older than 85 years increased significantly. The length of stay was significantly longer for patients older than 65 years than for younger patients. Ogawa et al. (2006) reported that factors of prolonged hospitalisation for elderly patients included complications and the worsening of other diseases, ${ }^{14}$ while Yoshida et al. (2016) observed the exacerbation of original diseases in $16 \%$ of elderly patients, physical complications after hospitalisation in $10.4 \%$ and physical problems in roughly $25 \% .{ }^{13}$

Because we analysed data using only the diagnosed diseases of patients, the potential involvement of post-stay complications remains unknown. Nevertheless, even patients older than 65 years who stayed for trauma exhibited several coexisting medical problems, including pneumonia, urinary tract infection and arrhythmia, which suggests that physical factors could have influenced the length of stay for elderly patients overall. Among patients older than 65 years, the maximum length of stay was 6 days, which allowed us to make policy-related decisions to, for example, transfer patients to other departments or hospitals or to discharge them in a relatively short time.

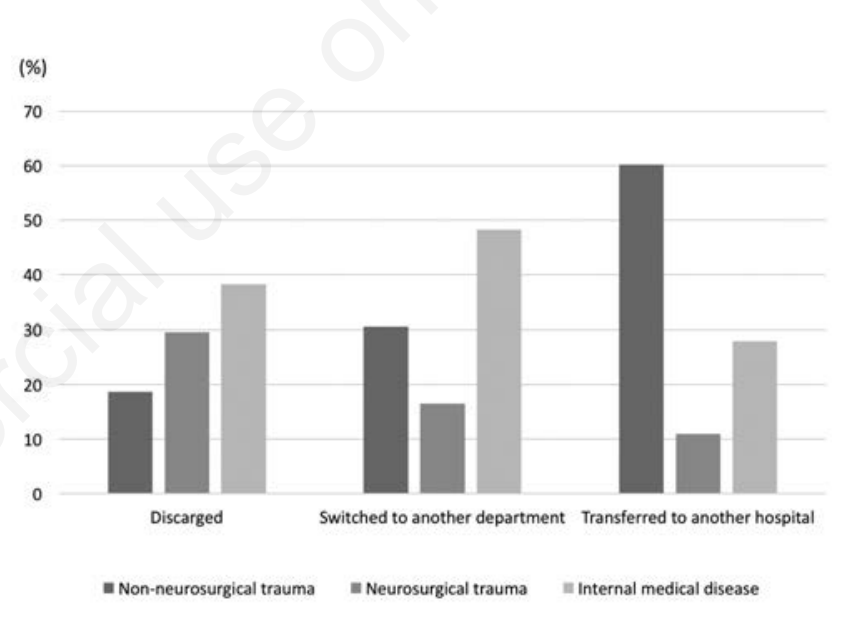

Figure 5. Details of the proportions of patients who were discharged, transferred to other departments, or transferred to other hospitals.

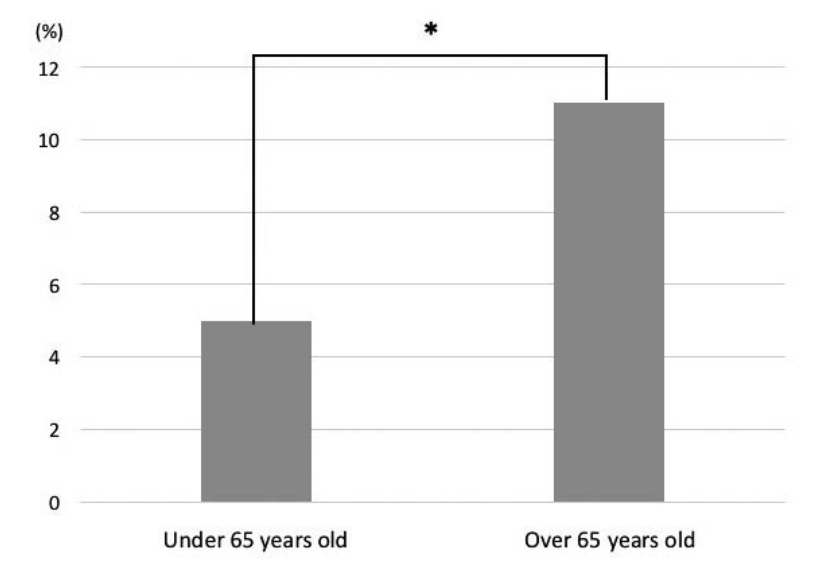

Figure 6. Rate of coexisting medical problems among patients who presented with trauma. We analysed data with a chi-square test $(\star P<.05)$. 
According to a 2013 survey by the Japanese Hospital Association, the average rate of refusal to accept ambulances at hospitals in Japan from 2010 to 2012 changed only slightly, from $18.1 \%$ in 2010 to $18.4 \%$ in 2011 to $18.1 \%$ in $2012 .{ }^{15}$ Major reasons for refusal included the absence of specialists or doctors capable of responding $(55.4 \%)$, that all doctors were busy with surgery or treatment $(49.1 \%)$ and that all beds were occupied $(26.5 \%) .9,15,16$ Whereas medical institutions with refusal rates of less than $10 \%$ (i.e. regional core hospitals, including critical emergency centres) accounted for less than $40 \%$ of all hospitals in the survey, medical institutions with rates of refusal exceeding 30\% slightly increased, from $22.6 \%$ in 2010 to $23.3 \%$ in 2011 to $24.8 \%$ in 2012 . Thus, it is possible that trends of refusing or accepting ambulances at Japan's emergency medical institutions is becoming increasingly polarised. ${ }^{15}$

In another survey by the Tokyo Metropolitan Welfare Bureau, the most prominent keywords describing patients categorised as difficult to place following ambulance transport (i.e. in adherence to the Tokyo Rule) fell into the categories of orthopaedics and elderly patients. Among patients eligible to use the Tokyo Rule, the proportion 76-89-year-olds was greatest (35.8\%), while patients older than 65 years accounted for $70.9 \%$ of all patients. ${ }^{9}$ The evidence suggests that patients seeking care in emergency departments are concentrated in emergency departments at regional core hospitals, where it is difficult to accommodate elderly patients with trauma or suspected of having orthopaedic disease.

As our results show, approximately $35 \%$ of conditions identified at the time of admission to the EDOU among patients older than 65 years were non-neurosurgical trauma, whereas $60 \%$ were other forms of trauma. Those proportions are significantly greater than proportions among patients younger than 65 years.

In the EDOU, the management of patients likely to be difficult to place definitively included initial treatment, diagnosis and shortterm admission. Most patients with orthopaedic disease prepped for surgery were transferred to other hospitals that could perform surgery within a few days. Space was also freed up in the emergency department by discharging patients directly to their homes and admitting patients requiring certain services to appropriate hospital departments or elsewhere. Those discharges and transfers improved the management of the overstretched local emergency system that like other systems has experienced staff shortages. However, in an ideal system, patients are treated from the start, in the right place and at the right time.

\section{Conclusions}

Our analysis of data of patients hospitalised in a university hospital's EDOU revealed that many elderly patients with trauma were processed in the EDOU. We thus suggest that deploying an EDOU for patients who can be hospitalised for several days in the emergency department of university hospitals can accommodate elderly patients with trauma who are likely to be difficult to accommodate otherwise. Overall, our results indicate that EDOUs at university hospitals are useful as buffers for overstretched regional emergency medical systems in Japan. Overcrowding at such facilities is likely to be an ongoing problem given the continued ageing of the Japanese population. However, our results do not indicate whether the strategy used is more efficient, both in terms of cost-effectiveness and clinical outcomes, than developing appropriate orthopaedic and trauma resources that would allow elderly patients to be directly admitted to corresponding departments.

\section{References}

1. Ministry of Internal Affairs and Communications Fire and Disaster Management Agency: Current status of emergency and rescue in 2012.

2. Fornero G, Arione R, Fiandra U, et al. Overcrowding in emergency departments: the case of the San Giovanni Battista (Molinette) University Hospital in Turin (Italy). Iq Sanità Pubbl 2011;67:541-52.

3. Kagobora P, Ntombifikile GM. A descriptive analysis of emergency department overcrowding in a selected hospital in Kigali, Rwanda. Afr J Emerg Med 2014;4:178-83.

4. Arrug W, Yuwares S, Pungkava S, et al. Factors associated with overcrowded emergency rooms in Thailand: a medical school setting. Emerg Med Inter 2014. Available from: http://dx.doi.org/10.1155/2014/576259

5. Emergency Promotion Foundation Survey Research Grant Project. Prospective study on the reality of emergency transportation acceptance refusal. 2009.

6. Ministry of Health, Labor and Welfare. The way of emergency medical system for elderly patients. 2013.

7. Ministry of Internal Affairs and Communications Fire and Disaster Management Agency. Current status of emergency and rescue in 2016. Available from: https://www.mhlw.go. jp/english/new-info/2016.html

8. United Nations Department of Economic and Social Affairs. World Population Prospects: 2015. Available from: http://www.un.org/en/development/desa/publications/worldpopulation-prospects-2015-revision.html

9. Tokyo Metropolitan Welfare Bureau. Emergency medical system to respond to changes in social structure. 2013.

10. Overcrowding crisis in our nation's emergency departments: is our safety net unraveling? Pediatrics 2004;114:878-88.

11. Gentile S, Vignally P, Durand AC, et al. Nonurgent patients in the emergency department? A French formula to prevent misuse. BMC Health Service Res 2010;10:66.

12. Moseley MG, Hawley MP, Caterino JM, et al. Emergency department observation units and the older patient. Clin Geriatr Med 2013;29:71-89.

13. Yoshida M, Ishikawa H, Yokoyama T, et al. Problems of emergency medical care for the elderly based on the data of patients hospitalized in emergency hospitals in Tokyo. J Jpn Assoc Acute Med 2015;26:687-701.

14. Ogawa T, Yuasa M, Ishizuka A, et al. Factor in long-term hospitalized in special geriatric hospital: case studies. J Health Care Nurs 2006;2:66-72.

15. Japan Hospital Association. Emergency medical questionnaire survey results in 2013. 2013.

16. Kamekura S, Kanai H, Kobayashi A, et al. Survey of the cases in which the needs of orthopaedic surgeries could not be accepted at our hospital. J Jpn Soc Emerg Med 2014;17:78993. 